\title{
Admission Heart Rate Variability is Associated with Depression and Cognition in Patients with Acute Mild-Moderate Ischemic Stroke
}

\section{Lanying he}

Second people's Hospital of Chengdu https://orcid.org/0000-0002-7127-6333

Ronghua Xu ( $\sim 2234117052 @ q q . c o m$ )

The Second People's Hospital of Chengdu

Jian Wang

The Second People's Hospital of Chengdu

Lili Zhang

The Second People's Hospital of Chengdu

Weiwei Dong

Chongqing Medical University

Hao Yang

Chongqing University

Research article

Keywords: Acute ischemic stroke; depression; cognitive; heart rate variability; fractal dimension;

Posted Date: May 13th, 2019

DOI: https://doi.org/10.21203/rs.2.9551/v1

License: (1) This work is licensed under a Creative Commons Attribution 4.0 International License.

Read Full License 


\section{Abstract}

Background Stroke has been shown to cause cardiac autonomic dysfunction. Depression and cognitive impairment are common complications after acute ischemic stroke (AIS). The relationship between poststroke depression (PSD) and cognitive impairment ( $\mathrm{PCl}$ ) and heart rate variability(HRV) was unclear. The purpose of this study was to investigate whether the decreased HRV was related to PSD and PCI in patients with mild-moderate AIS. Methods Changes in HRV after AIS were assessed using the nonlinear fractal dimension (FD) method, and patients within 72 hours of AIS were included in the study. 476 patients were included in this study. All patients underwent mood tests, cognitive test at 3 months. Cognitive and mood state were assessed using the Montreal Cognitive Assessment (MoCA) and the 15item Stroke Specific Geriatric Depression Scale (GDS), respectively. PSD was defined if GDS $\geq 5$ and PSCl was defined if MoCA<26. We assessed the relationship between FD and PSD and PSCl at 3 months. Results 50.84\% (242/476) of patients had PSD, and 33.19\% (158/476) of patients had PSCl. Compared with no PSD group, the lower NIHSS and FD value,and higher prevalence of FD $\leq 1.05$ were more likely in patients with PSD $(P<0.05)$. Compared with no PSCl group, the higher prevalence of $F D \leq 1.05$ were more likely in patients with $\mathrm{PSCl}(\mathrm{P}<0.05)$.In fully adjusted models, the $\mathrm{FD} \leq 1.05$ was significantly associated with PSD (adjusted OR, 3.31; 95\% Cl, 1.81-5.43; $\mathrm{P}=0.000$ ), and $\mathrm{PSCl}$ (adjusted OR,1.88; 95\% $\mathrm{Cl}, 1.11-3.16$; $P=0.018$ ). Conclusions These results suggested that $F D \leq 1.05$ after AIS could be used as an objective tool for early prediction of PSD and PSCl, providing guidance for the treatment of PSD and PCl, and improving the prognosis of patients.

\section{Background}

Stroke was the most important disabling and fatal disease in China [1]. Stroke could lead to physical disability, cognitive and emotional impairments. Post-stroke depression(PSD) was very common after stroke. Previous studies had shown that the prevalence in the first year after stroke was about $41.8 \%[2]$. PSD might be caused by physical disability [3]. PSD had negative effects on the recovery, mortality, quality of life, physical and cognitive functions of stroke patients[4-6].

There were many reports about cognitive impairment after stroke $[7,8]$. Post-stroke cognitive impairment (PSCl) could lead to increased risk of death, decreased functional recovery, and evolve to dementia[9-11]. Over time, there appeared to be a complex interaction between depressive symptoms and cognitive function in stroke patients. Cognitive impairment and depressive symptoms might overlap each other $[13,14]$, and both might coexist in stroke patients $[15,16]$, cognitive impairment might also be caused by depression [17].

It had been reported that stroke could cause cardiac autonomic dysfunction and heart rate variability (HRV) decreased, which reflecting poor parasympathetic regulation. Decreased HRV was also related to depression [19] and poor cognitive function [20]. Because healthy heart rates were slightly irregular and chaotic, chaos theory could better explain the dynamics of heart rate. In physiological and pathological conditions, nonlinear methods could provide new ideas for HRV research [21-23]. FD was a characteristic 
parameter in chaos theory, which could quantify the complexity of HRV and was one of the most commonly used nonlinear methods. FD method had the advantages of high precision and simple calculation. FD was related to dimensional complexity, and it estimates the self-similarity of a time interval in time series [24].

The purpose of this study was to discuss whether the reduction of FD was related to depression and cognitive function after mild-moderate AIS.

\section{Methods}

\section{Study Population}

Patients with mild-moderate acute ischemic stroke(AIS) within 72 hours of onset were selected. All patients met the WHO diagnostic criteria, were confirmed by brain CT or diffusion weighted imaging (DWI) magnetic resonance imaging (MRI). Eligible patients were admitted to our stroke ward. The severity of stroke was assessed by the the National Institutes of Health Stroke (NIHSS) score. All study participants or their legal representatives agreed to participate in the trial and they also signed informed consent. This study was approved by the local ethics committee.

\section{Inclusion criteria}

Only patients who met all of the following criteria were included in the study: (1). Aged from 18-80 years old; (2) Acute ischemic stroke occurred for the first time within 72hours after onset; (3) The lesion was single and related to clinical manifestations; (4). Finnish-speaking; (5). Able to co-operate; (6). NIHSS score $\leq 8 ;(7)$. All patients were not treated with intravenous thrombolysis or mechanical thrombolysis. All patients received standard medical treatment, including aspirin and lipid-lowering drugs.

\section{Exclusion criteria}

(1) cerebral hemorrhage, cardiogenic stroke, Brainstem or cerebellar stroke; (2) pre-existing neurological or psychiatric diseases that affect cognitive function; (3). Patients with severe heart disease and various severe arrhythmias (such as acute myocardial infarction, history of tachycardia/tachycardia or atrial fibrillation), diabetes with disease duration longer than 5 years and evidence of neuropathy; (4). Severe lung disease, renal failure (estimated glomerular filtration rate $₫ 30 \mathrm{ml} / \mathrm{min} .1 .73 \mathrm{~m}^{2}$ ), and active malignancies; (5) aphasia or severe hearing impairment; (6), fever $\left(38^{\circ} \mathrm{C}\right.$ or higher), hypoxemia (arterial oxygenation hemoglobin saturation $<90 \%)$; (7) drug or alcohol abuse. All patients were followed up for 3 months. 


\section{Data Collection and Scale Assessment}

Detection of 12-lead ECG and HRV in the next morning 9:00 to 10:00 AM, patients with relaxation supine state, in the quiet environment, the room temperature was about $22^{\circ} \mathrm{C}$.

The ECG sampling frequency was $1000 \mathrm{hz}$ and required 15 minutes to obtain continuous R-R interval sequences, about 2,000 beats. The R-R interval sequences were passes through a filter to eliminate interfering factors, such as noise, artifacts, and premature beats. All R-R interval sequences were automatically edited at first, and then carefully edited manually. Excluding the interfering part, only the part of $>90 \%$ pure sinus beats were included in the analysis. Finally, 512 continuous R-R interval sequences were selected for HRV analysis.

The chaotic characteristics of R-R sequences were represented by FD, which was used to quantify the complexity of the R-R dynamic changes, $F D$ equation was $F D=\log N(\varepsilon) / \log (1 / \varepsilon), \varepsilon$ was the range, which was used to monitor the R-R interval, and $N(\varepsilon)$ was the number of the R-R intervals [29]. FD parameters of each subject were calculated automatically by off-line computer software. Decreased FD was defined as $\mathrm{FD} \leq 1.05$ according to our previous studies $[30,31]$.

Depression was assessed 3 months later. The patients were assessed by an experienced neurologist who blinded to the clinical study and the patient's clinical information. Depression was assessed using the 15item Stroke Specific Geriatric Depression Scale (GDS), it consists of 15 questions with a total of 15 points. Diagnostic criteria: 0-4, no depression; 5-8, mild depression; 9-11, moderate depression; 12-15, severe depression.

The Montreal Cognitive Assessment (MoCA) was used for the assessment of cognitive impairment, with a range of 0 to 30 points. A lower MoCA score indicates more severe cognitive impairment, and a score $<26$ was considered to be cognitive impairment.

\section{Statistical Analysis}

Patients were divided into PSD and PSD groups, PSCl and PSCl groups. Demographic characteristics, vascular risk factors, current smoking, and so on were compared between two groups in univariate analysis, distributions of continuous variables were determined by the Kolmogorov-Smirnov test, while Mann-Whitney two sample test was applied in case of non-normal distributions. We used Pearson $\chi 2$ test, Fisher exact 2-sided test, or Student t test for data analyze. Adjusting for all confounders (such as age, baseline NIHSS score, gender, BMI, hypertension, current smoking, diabetes, hyperlipidemia, insular stroke, family history of stroke, etiology, and drug use), multivariate logistic regression was used to analyze the relationship between FD and outcomes (PSD, PSCI). The adjusted odds ratio (OR) and 95\% confidence interval $(\mathrm{Cl})$ represent the analysis results. SPASS 22.0 software was used for all data analyze. $P<0.05$ was considered as statistically significant. 


\section{Results}

\section{Characteristics of the study subjects}

A total of 476 patients were included in the study, including $48.95 \%(233)$ males and $51.05 \%(243)$ females, with an average age of $65.88 \pm 10.14$ years (39-80 years), there were 313 patients with hypertension, 144 with diabetes, 235 with hyperlipidemia, and 127 with smoking. The mean NIHSS score was $6.55( \pm 1.93)$. During the 3-month follow-up, no patients died and no patients No patients were lost to follow-up.

\section{Univariable Models for Predictors of PSD}

242 (50.84\%) patients had PSD. The comparison of basic characteristics between PSD group and PSD group was shown in table 1. Age distribution, gender distribution, BMI, hypertension and diabetic prevalence, lipid profiles, and so on were similar between these 2 groups. Patients with PSD showed significantly lower NIHSS $(6.12 \pm 2.16$ vs $6.69 \pm 1.85 ; P=0.007)$ than patients with no PSD. Compared with the PSD group, the FD value in the PSD group was lower ( $1.33 \pm 0.36$ vs $1.41 \pm 0.32, P=0.001)$, and the prevalence of $F D \leq 1.05$ was higher $(26.03 \%$ vs $10.68 \%, P=0.000)$.

\section{Univariable Models for Predictors of PSCl}

$\mathrm{PSCl}$ occurred in 158 patients (33.19\%) at 3 months. At baseline, age and gender distribution, $\mathrm{BMI}$, the prevalence of hypertension, diabetes mellitus, and blood lipid profile, and soon were similar between the two groups (table 2). Compared with no PSCl group, the prevalence of FD $\leq 1.0$ in PSCl was higher1.05(25.68\%vs $15.41 \%, P<0.014)$. However, there was no significant difference in FD value between the two groups.

\section{Multivariable Models on the Association between FD $\leq 1.05$ and PSD, PSCI}

There was an association between FD $\leq 1.05$ and PSD (OR,2.94, 95\%Cl, 1.78-4.87, $\mathrm{P}=0.000)$, and with PSCl $(\mathrm{OR}, 1.90,95 \% \mathrm{Cl}, 1.12-2.89, \mathrm{P}=0.014)$ in unadjusted models. In the multivariable logistic regression model after adjustment for confounding factors, FD $\leq 1.05$ was significant predictor for PSD (adjusted OR, 3.31; 95\% Cl, 1.81-5.43; $\mathrm{P}=0.000$ ), and $\mathrm{PSCl}$ (adjusted OR,1.88; 95\% Cl, 1.11-3.16; $\mathrm{P}=0.018$ ) (Table 3), after controlling for confounding factors, NIHSS was associated with PSD (adjusted OR,1.20; 95\% Cl, 1.091.32; $\mathrm{P}=0.000)$, but $\mathrm{PSCl}$ was not significantly associated with NIHSS. 


\section{Discussion}

Cardiac dysautonomia was a common complication of stroke, HRV analysis was a common tool for studying cardiac autonomic control. In previous studies, linear statistical methods (time-domain and frequency-domain methods) were usually used to analyze HRV, which detected the overall amplitude of RR interval fluctuation around its mean value [32]. However, it provided very limited HRV information because the nonlinear mechanism seems to be involved in the origin of heart rate dynamics [33].

FD was one of the most common nonlinear parameters in chaotic characteristics, and could quantify the complexity of HRV [24]. FD algorithm estimated the self-similarity of a time interval in time series, which was related to the complexity of time series. In this study, FD was used to evaluate the status of autonomic function of AIS.

HRV had been the focus of research on biomarkers for depression and cognition after AIS in the last couple of decades [34], only the traditional time linear method was considere[35,36]. In this study, we investigated the relationship between FD, depression and cognition after AIS

Our results showed significant differences between groups of patients with PSD and no PSD. Lower FD values and the higher prevalence of FD $\leq 1.05$ in PSD group compared to no PSD group. This result might be due to the reduced ability of the parasympathetic nervous system to regulate heart rate through vagal activity [37,38], and the reduced short-term flexibility of ANS to adapt to environmental and task changes [39-41].

Interestingly, we also founded that the incidence of decreased FD $\leq 1.05$ was higher in the PSCl groups, but FD value in early poststroke phase did not differ in the two groups. It was possible that because of the subject we studied, stroke patients are mild-moderate acute ischemic stroke, and the low incidence of $\mathrm{PSCl}$ in the mild-moderate stroke. This study showed that cognitive impairment was more likely in patients with $F D \leq 1.05$. The results of this study support the relationship between low vagal tone and poor cognitive function. The prediction of cognitive function by the HR parameters measured could be explained by the well-known effect of post-stroke depression on cognitive function in early phase. However, it had recently been reported that reduced HRV at rest was associated with reduced whole-brain perfusion, which itself was associated with an increased risk of post-stroke dementia. Therefore, the effect of decreased HRV on chronic cognitive function may be mediated by cerebral perfusion insufficiency $[42,43]$.

After adjusting for fully confounders, the results showed that there were significant association of FD $\leq 1.05$ with risks of 3-month PSD and PSCI after AIS. To conclude, This study found that FD measurement was helpful in predicting PSD and PSCI in the early stage of stroke, especially in the case of severe aphasia or cognitive impairment, in which questionnaire could not be used.

However, due to some limitations, these results must be carefully interpreted and could not be generalized to all stroke patients. First, the patients were mild-moderate stroke, and severe stroke was excluded. 
Second, the absence of FD measurements at the 3-month, which did not clarify the causal relationship between cardiac autonomic function, depression, and cognitive impairment after stroke. Third, although we adjusted the NIHSS score, the NIHSS score was related to the infarct volume, we did not measure the infarct volume in this study. Fourth, previous studies had shown that bilateral insular lesions might have different effects on cardiac autonomic function. We did not analyze the possible effects of bilateral insular stroke on FD, PSD and PSCI. In addition, larger multicenter clinical studies should be needed to confirm our results, which may provide a better understanding of the pathophysiological mechanisms between poststroke autonomic function, cognitive impairment, and mood disorders. However, despite these limitations, the advantage of our study lies in its large sample size, the adjustment of various confounders in its analysis, and the use of standardized methods to measure FD.

\section{Conclusions}

In summary, our findings suggested that decreased FD in AIS was associated with an increased risk of PSD and PSCl at 3 months. Low HRV may be a sign of higher susceptibility to PSD and PSCI. FD has potential predictive value for PSD and PSCl after ischemic stroke.

\section{Abbreviations}

Cl: Confidence Interval; M: Mean; OR: Odds Ratio; SD: Standard Deviation; FD: fractal dimension; PSD:Poststroke depression; AIS:acute ischemic stroke; HRV: heart rate variability; PSCl: Post-stroke cognitive impairment;

\section{Declarations}

\section{Ethics approval and consent to participate}

This study was approved by the the Medical and Health Research Ethics Committee of the Second people's Hospital of Chengdu. The present study was carried out on the Helsinki declaration. This study followed the regulations of local laws and regulatory agencies to keep patients' medical information confidential. Prior to registration, the physician provided each patient or their legal representative with detailed information about the purpose, scope, and possible consequences of the trial. Clinical trials do not require intervention. All patients or their close relatives signed the consent form.

\section{Consent for publication}

Not applicable.

\section{Availability of data and materials}

The datasets used and/or analyzed during the current study are available from the corresponding author on reasonable request. 


\section{Competing interests}

The authors declare that they have no competing interests.

\section{Funding}

This work was funded by the Health and Family Planning Commission of Chengdu (2015009), which is not involved in the database management (collection, analysis, interpretation of data) and has no access to patient information. The funding body did not participate in designing the study or writing the manuscript. The study protocol has undergone peer-review process by the funding body.

\section{Authors' contributions}

LYH was responsible for data collection and analysis, as well as the writing of the first draft and subsequent drafts of the paper. RX and JW were responsible for the design and interpretation of the study. LLZ was responsible for data analysis. WD was responsible for the conceptual interpretation of the study, and HY was responsible for the design of FD off-line calculation software. All authors read and approved the final manuscript for publication

\section{Acknowledgments}

We thank all patients and their families for generously consenting to use of human tissues in this research.

\section{References}

1.Wang W, Jiang B, Sun H2, Ru X, Sun D, Wang L, Wang L, Jiang Y, Li Y, Wang Y, Chen Z, Wu S, Zhang Y, Wang D, Wang Y, Feigin VL; Prevalence, Incidence, and Mortality of Stroke in China: Results from a Nationwide Population-Based Survey of 480687 Adults. Circulation. 2017;135:759-771. doi: 10.1161/CIRCULATIONAHA.116.025250.

2. Zhang N, Wang CX, Wang AX, Bai Y, Zhou Y, Wang YL, et al. Time Course of Depression and One-Year Prognosis of Patients with Stroke in Mainland China. CNS Neurosci Therapeut. 2012; 18:475-481. doi: 10.1111/j.1755-5949.2012.00312.x

3. Ayerbe L, Ayis SA, Crichton S, Rudd AG, Wolfe CD. Explanatory factors for the association between depression and long-term physical disability after stroke. Age Ageing. 2015;44:1054-1058. doi: 10.1093/ageing/afv132

4. Karaahmet OZ, Gurcay E, Avluk OC, Umay EK, Gundogdu I, Ecerkale O, et al. Poststroke depression: risk factors and potential effects on functional recovery. Int J Rehabil Res. 2017;40:71-75. doi: 10.1097/MRR.0000000000000210

5. Shi YZ, Xiang YT, Yang Y, Zhang N, Wang S, Ungvari GS, et al. Depression after minor stroke: the association with disability and quality of life-a 1-year follow-up study. Int J Geriat 
Psychiatry.2016;31:421-427. doi: 10.1002/gps.4353

6. Guajardo VD, Terroni L, Sobreiro Mde F, Zerbini MI, Tinone G, Scaff M, et al. The influence of depressive symptoms on quality of life after stroke: a prospective study. J Stroke Cerebrovasc Dis.2015;24:201-209.doi: 10.1016/j.jstrokecerebrovasdis.2014.08.020.

7. Hoffmann M, Schmitt F, Bromley E. Vascular cognitive syndromes: relation to stroke etiology and topography. Acta Neurol Scand. 2009;120:161-9. doi: 10.1111/j.1600-0404.2008.01145.x

8. SunN, LiQ-J, LvD-M,Man J, Liu X-S, SunM-L. A survey on 465 patients with post-stroke depression in China. Arch Psychiatric Nurs. 2014; 28:368-371. doi: 10.1016/j.apnu.2014.08.007

9. Barker-Collo S, Feigin V. The impact of neuropsychological deficits on functional stroke outcomes. Neuropsychol Rev.2006;16:53-64. doi: 10.1007/s11065-006-9007-5

10. Farner L, Wagle J, Engedal K, Flekkøy KM, Wyller TB, Fure B. Depressive symptoms in stroke patients: a 13month follow-up study of patients referred to a rehabilitation unit. J Affect Disord.2010;127:211-218. doi: 10.1016/j.jad.2010.05.025

11. Hobson P, Meara J. Cognitive function and mortality in a community-based elderly cohort of first-ever stroke survivors and control subjects. J Stroke Cerebrovasc Dis. 2010;19:382-387.doi: 10.1016/j.jstrokecerebrovasdis.2009.07.006

12. Terroni L, Sobreiro MF, Conforto AB, Adda CC, Guajardo VD, Lucia MCSd, et al. Association among depression, cognitive impairment and executive dysfunction after stroke. Dement Neuropsychol. 2012;6:152-157. doi: 10.1590/S1980-57642012DN06030007ロ

13. Murata Y, Kimura M, Robinson RG. Does cognitive impairment cause poststroke depression? Am J Geriat Psychiatry.2000;8:310-317. doi: 10.1097/00019442-200011000-00007

14. Rose E, Ebmeier K. Pattern of impaired working memory during major depression. J Affect Disord. 2006;90:149-161. doi: 10.1016/j.jad.2005.11.003

15. Elbaz A, Vicente-Vytopilova P, Tavernier B, Sabia S, Dumurgier J, Mazoyer B, et al. Motor function in the elderly Evidence for the reserve hypothesis. Neurology. 2013;81:417-426. doi: 10.1212/WNL.0b013e31829d8761

16. Pendlebury ST, Rothwell PM. Prevalence, incidence, and factors associated with pre-stroke and poststroke dementia: a systematic review and meta-analysis. Lancet Neurol.2009;8:1006-1018. doi: 10.1016/S1474-4422(09)70236-4

17. Kauhanen M-L, Korpelainen J, Hiltunen P, Brusin E, Mononen H, Määttä R, et al. Poststroke depression correlates with cognitive impairment and neurological deficits. Stroke.1999;30:18751880. doi: 10.1161/01.STR.30.9.1875

18. Dütsch M, Burger M, Dörfler $C$, et al. Cardiovascular autonomic function in poststroke patients. Neurology. 2007;69:2249-2255.

19. Kemp AH, Quintana DS, Gray MA, et al. Impact of depression and antidepressant treatment on heart rate variability: a review and meta-analysis. Biol Psychiatry.2010;67:1067-1074. 
20. Thayer JF, Hansen AL, Saus-Rose E, et al. Heart rate variability, prefrontal neural function, and cognitive performance: the neurovisceral integration perspective on self-regulation, adaptation, and health. Ann Behav Med.2009;37:141-153.

21. Goldberger AL, Amaral LA, Hausdorff JM, Ivanov PCh, Peng CK, Stanley HE.Fractal dynamics in physiology: alterations with disease and aging. Proc Natl Acad Sci U S A .2002;99 Suppl 1:2466-72. doi:10.1073/pnas.012579499.

22. Lombardi, F. Chaos theory, heart rate variability, and arrhythmic mortality. Circulation.2000;10:8-10. PMID:10618296

23. Peng, C. K., Costa, M. \& Goldberger, A. L. ADAPTIVE DATA ANALYSIS OF COMPLEX FLUCTUATIONS IN PHYSIOLOGIC.TIME SERIES. Adv Adapt Data Anal.2009;1:61-70.

doi:10.1142/S1793536909000035. PMID:20041035

24. Higuchi T. Approach to an irregular time series on the basis of the fractal theory. Physica $D(1988) ; 31$ : 277-283.

25. He L, Li C, Luo Y, Dong W, Yang H. Clinical prognostic significance of heart abnormality and heart rate variability in patients with stroke. Neurol Res.2010;32:530-534. doi:

10.1179/174313209X431110. PMID:19473556

26. Mäkikallio AM, Mäkikallio TH, Korpelainen JT, Sotaniemi KA, Huikuri HV, Myllylä VV. Heart rate dynamics predict poststroke mortality. Neurology.2004;62:1822-1826. PMID:15159485

27. Colivicchi F, Bassi A, Santini M, Caltagirone C. Cardiac autonomic derangement and arrhythmias in right-sided stroke with insular involvement. Stroke.2004;35:2094-2098.

doi:10.1161/01.STR.0000138452.81003.4c. PMID:15272134

28. Lin DC, Sharif A. Common multifractality in the heart rate variability and brain activity of healthy humans. Chaos.2010;20:023121. doi: 10.1063/1.3427639. PMID:20590317

29. Xu Xiaohong, Xie Zhenxiang, Chen Liangchi. A computerized system for analysing chaotic characteristics of heart period signal. Chinese J Bio Med Eng.1999;18:74-88.

30. Xu XH, Xie Zhenxiang, Chen Liangchi. A computerized system for analysing chaotic characteristics of heart period signal. Chinese J Bio Med Eng. 1999;18:74-88.

31. He L, Li C, Luo Y, Dong W, Yang H. Clinical prognostic significance of heart abnormality and heart rate variability in patients with stroke. Neurol Res.2010;32:530-534. doi: 10.1179/174313209X431110.

32. He L, Wang J, Zhang L, Zhang X, Dong W, Yang H.Decreased fractal dimension of heart rate variability is associated with early neurological deterioration and recurrent ischemic stroke after acute ischemic stroke. J Neurol Sci. 2019 Jan 15;396:42-47. doi: 10.1016/j.jns.2018.11.006. Epub 2018 Nov 5

33. Yperzeele L, van Hooff RJ, Nagels G, De Smedt A, De Keyser J, Brouns R. Heart rate variability and baroreceptor sensitivity in acute stroke: a systematic review. Int J Stroke. 2015;10:796-800. doi: 10.1111/ijs.12573. Review. 
34. Tessier A, Sibon I, Poli M, Audiffren M, Allard M, Pfeuty M.Resting Heart Rate Predicts Depression and Cognition Early after Ischemic Stroke:A Pilot Study. J Stroke Cerebrovasc Dis. 2017;26:2435-2441. doi: 10.1016/j.jstrokecerebrovasdis.2017.05.040.

35. Hartmann R, Schmidt FM, Sander C, Hegerl U. Heart Rate Variability as Indicator of Clinical State in Depression. Front Psychiatry. 2019:735. doi: 10.3389/fpsyt.2018.00735.

36. Sgoifo A, Carnevali L, Alfonso Mde L, Amore M.Autonomic dysfunction and heart rate variability in depression. Stress. 2015;18:343-352. doi: 10.3109/10253890.2015.1045868. Epub 2015 May 25.

37. Carreno FR, Frazer A. Vagal nerve stimulation for treatment resistant depression. Neurotherapeutics. 2017;14:716-727. doi: 10.1007/s13311-017-0537-8

38. Aaronson ST, Sears P, Ruvuna F, Bunker M, Conway CR, Dougherty DD, et al. A 5-Year observational study of patients with treatmentresistant depression treated with vagus nerve stimulation or treatment as usual: comparison of response, remission, and suicidality. Am J Psychiatry.2017;174:640-648. doi: 10.1176/appi.ajp.2017.16010034

39. Williams DP, Cash C, Rankin C, Bernardi A, Koenig J, Thayer JF. Resting heart rate variability predicts self-reported difficulties in emotion regulation: a focus on different facets of emotion regulation. Front Psychol.2015;6:261. doi: 10.3389/fpsyg.2015.00261

40. Appelhans BM, Luecken LJ. Heart rate variability as an index of regulated emotional responding. Rev Gen Psychol.2006;10:229-240. doi: 10.1037/1089-2680.10.3.229

41. Sakaki M, Yoo HJ, Nga L, Lee TH, Thayer JF, Mather M. Heart rate variability is associateed with amygdala functional connectivity with MPFC across younger and older adults. Neurolmage.2016;139:44-52. doi: 10.1016/j.neuroimage.2016.05.076

42. Allen $B$, Jennings JR, Gianaros PJ, et al. Resting highfrequency heart rate variability is related to resting brain perfusion. Psychophysiology.2015;52:277-287.

43. Desmond DW, Moroney JT, Sano M, et al. Incidence of dementia after ischemic stroke results of a longitudinal study. Stroke.2002;33:2254-2262.

\section{Tables}

Table 1. Comparison of baseline characteristics between patients with no PSD and PSD groups. 


\begin{tabular}{lllll}
\hline & no PSD group (234) & PSD group (242) & OR(95\%Cl) & $\mathrm{P}^{*}$ \\
\hline Age, $\mathrm{y}($ Mean SD) & $66.01 \pm 10.34$ & $65.74 \pm 9.96$ & & 0.767 \\
NIHSS score(Mean SD) & $6.69 \pm 1.85$ & $6.12 \pm 2.16$ & & 0.007 \\
FD (Mean SD) & $1.41 \pm 0.32$ & $1.33 \pm 0.36$ & & 0.001 \\
FD $\leq 1.05, \mathrm{n}(\%)$ & $25(10.68)$ & $63(26.03)$ & $2.94(1.78-4.87)$ & 0.000 \\
Females,n(\%) & $118(50.43)$ & $125(51.65)$ & $1.05(0.73-1.51)$ & 0.789 \\
Men,n(\%) & $116 \square 49.57 \square$ & $117(48.35)$ & $1.05(0.73-1.51)$ & 0.789 \\
BMl $\geq 24 \mathrm{~kg} / \mathrm{m}, \mathrm{n}(\%)$ & $64(27.35)$ & $76(31.40)$ & $1.22(0.82-1.81)$ & 0.332 \\
Hypertension,n(\%) & $163(69.66)$ & $150(61.98)$ & $0.71(0.49-1.04)$ & 0.078 \\
Current smoking,n(\%) & $62(26.50)$ & $65(26.86)$ & $1.02(0.68-1.53)$ & 0.929 \\
Diabetes, $\mathrm{n}(\%)$ & $77(32.91)$ & $67(27.69)$ & $0.78(0.53-1.16)$ & 0.215 \\
Hyperlipidemia,n(\%) & $109(46.58)$ & $126(52.07)$ & $1.25(0.87-1.79)$ & 0.231 \\
Insular stroke,n(\%) & $47(20.09)$ & $55(22.73)$ & $1.17(0.75-1.82)$ & 0.483 \\
Family history of stroke,n(\%) & $44(18.80)$ & $47(19.42)$ & $1.04(0.66-1.64)$ & 0.864 \\
Etiological classification & & & & \\
Large artery atherosclerosis,n(\%) & $96(41.03)$ & $118(48.76)$ & $1.36(0.95-1.97)$ & 0.090 \\
Lacunar, $\mathrm{n}(\%)$ & $91(38.89)$ & $75(30.99)$ & $0.71(0.48-1.03)$ & 0.071 \\
Other known causes, $\mathrm{n}(\%)$ & $2(0.85)$ & $5(2.07)$ & $2.45(0.47-12.74)$ & 0.272 \\
Undetermined, $\mathrm{n}(\%)$ & $48(20.51)$ & $42(17.36)$ & $0.81(0.51-1.29)$ & 0.379 \\
Medications use & & & \\
Antiplatelet, $\mathrm{n}(\%)$ & $59(25.21)$ & $57(23.55)$ & $0.91 \square 0.60-1.39 \square$ & 0.673 \\
Antihypertensive, $\mathrm{n}(\%)$ & $139(59.40)$ & $124(51.24)$ & $0.72(0.50-1.03)$ & 0.073 \\
Lipid-lowering medications, $\mathrm{n}(\%)$ & $83(35.47)$ & $97(40.08)$ & $1.22 \square 0.84-1.76 \square$ & 0.300 \\
\hline
\end{tabular}

*Comparison between no PSD and PSD groups. Continuous variables are expressed as mean \pm standard deviation(SD). Categorical variables are expressed as frequency(percent) for $P$ values, Pearson $\chi 2$ test, Fisher exact 2-sided test, and Student $t$ test were used when appropriate. Distributions of continuous variables were determined by the Kolmogorov-Smirnov test, Mann-Whitney two sample test was applied in case of non-normal distributions.

Table 2. Comparison of baseline characteristics between patients with no PSCl and PSCl groups. 


\begin{tabular}{lllll}
\hline & no PSCl & PSCl group (158) & OR(95\%Cl) & P* \\
& group [318) & & & \\
\hline Age, y (Mean SD) & $65.69 \pm 10.12$ & $66.26 \pm 10.20$ & & 0.561 \\
NIHSS score (Mean SD) & $6.51 \pm 1.97$ & $6.63 \pm 1.85$ & & 0.724 \\
FD (Mean SD) & $1.39 \pm 0.34$ & $1.36 \pm 0.36$ & & 0.531 \\
FD $\leq 1.15, \mathrm{n}(\%)$ & $49(15.41)$ & $39(24.68)$ & $1.80(1.12-2.89)$ & 0.014 \\
Females, $\mathrm{n}(\%)$ & $169(53.14)$ & $74(46.84)$ & $0.78(0.53-1.14)$ & 0.195 \\
Men, $\mathrm{n}(\%)$ & $149 \square 46.86 \square$ & $84(53.16)$ & $0.78(0.53-1.14)$ & 0.195 \\
BMl $\geq 24$ kg/m, $\mathrm{n}(\%)$ & $101(31.76)$ & $39(24.68)$ & $0.70(0.46-1.08)$ & 0.111 \\
Hypertension, $\mathrm{n}(\%)$ & $206(64.78)$ & $107(67.72)$ & $1.14(0.76-1.71)$ & 0.524 \\
Current Smoking, $\mathrm{n}(\%)$ & $79(24.84)$ & $48(30.38)$ & $1.32(0.86-2.02)$ & 0.198 \\
Diabetes, $\mathrm{n}(\%)$ & $93(29.25)$ & $51(32.28)$ & $1.15(0.76-1.74)$ & 0.498 \\
Hyperlipidemia, $\mathrm{n}(\%)$ & $159(50.00)$ & $76(48.10)$ & $0.93(0.63-1.34)$ & 0.696 \\
Insular stroke, $\mathrm{n}(\%)$ & $73(22.96)$ & $29(18.35)$ & $0.75(0.47-1.22)$ & 0.249 \\
Family history of stroke, $\mathrm{n}(\%)$ & $57(17.92)$ & $34(21.52)$ & $1.26(0.78-2.02)$ & 0.348 \\
Etiological classification & & & & \\
Large artery atherosclerosis, $\mathrm{n}(\%)$ & $139(43.71)$ & $75(47.47)$ & $1.16(0.79-1.71)$ & 0.438 \\
Lacunar, $\mathrm{n}(\%)$ & $115(36.16)$ & $51(32.28)$ & $0.84(0.56-1.26)$ & 0.402 \\
Other known causes, $\mathrm{n}(\%)$ & $4(1.26)$ & $3(1.90)$ & $1.52(0.34-6.87)$ & 0.584 \\
Undetermined, $\mathrm{n}(\%)$ & $61(19.18)$ & $29(18.35)$ & $0.95(0.58-1.55)$ & 0.828 \\
Medications use & & & & \\
Antiplatelet, $\mathrm{n}(\%)$ & $70(22.01)$ & $46(29.11)$ & $1.46(0.94-2.25)$ & 0.089 \\
Antihypertensive, $\mathrm{n}(\%)$ & $171(53.77)$ & $92(58.23)$ & $1.20(0.82-1.76)$ & 0.357 \\
Lipid-lowering medications, $\mathrm{n}(\%)$ & $122(38.36)$ & $58(36.71)$ & $0.93(0.63-1.38)$ & 0.726 \\
\hline
\end{tabular}

*Comparison between no PSCl and PSCl groups. Continuous variables are expressed as mean \pm standard deviation. Categorical variables are expressed as frequency (percent) for $P$ values, Pearson $\chi 2$ test, Fisher exact 2-sided test, and Student $t$ test were used when appropriate. Distributions of continuous variables were determined by the Kolmogorov-Smirnov test, Mann-Whitney two sample test was applied in case of non-normal distribution.

Table 3 Multivariable Models Showing Association Between FD $\leq 1.05$ and Prognosis

\begin{tabular}{lrl}
\hline & OR $(95 \% \mathrm{Cl})$ & $\mathrm{P}^{*}$ \\
\hline $\mathrm{PSCl}$ & $1.88(1.11-3.16 \square$ & $\mathbf{0 . 0 1 8}$ \\
$\mathrm{PSD}$ & $3.31 \square 1.81-5.43 \square$ & $\mathbf{0 . 0 0 0}$ \\
\hline
\end{tabular}

*Multivariable adjusted for age, baseline NIHSS score, sex, BMI, hypertension, current smoking, current alcohol drinking, diabetes, hyperlipidemia, insular stroke, family history of stroke, etiological classification, medications use. 
Page 14/14 